\title{
LIX. Radio-active products of short life
}

\section{H.G.J. Moseley B.A. \& K. Fajans Ph.D.}

To cite this article: H.G.J. Moseley B.A. \& K. Fajans Ph.D. (1911) LIX. Radio-active products of short life , Philosophical Magazine Series 6, 22:130, 629-638, DOI: 10.1080/14786441008637158

To link to this article: http://dx.doi.org/10.1080/14786441008637158

曲 Published online: 20 Apr 2009.

Submit your article to this journal $\pi$

Џ Article views: 8

Q View related articles $₫$

4 Citing articles: 8 View citing articles 진 
a successive product of the group thorium $\mathrm{C}$ without any assumption whether it originates from only one of the components or both. The analogy between the general modes of the three emanations is undoubterlly very close. It appears very probable that the group radium $\mathrm{C}$ in reality corresponds to the group thorium $C+D$, and actinium $C+D$, although the type and order of the transformations appear to be different in each case. There are, however, still many questions to be closely examined before any decisive conclusion can be reached. The analogy between the products of the emanations has been discussed in some detail partly to give reasons for the scheme of nomenclature adopted, and partly to bring out the numerous points of similarity.

University of Manchester, August 1011 .

LIX. Radio-active Products of Short Life.

By H. G. J. Moseley, B.A., and K. Fajans, Ph.D.*

THE ordinary methods by which the properties of radio1 active substances are investigated are not suited to the study of products of short life. The shortest period hitherto measured, that of actinium emanation, period 3.9 seconds, was determined by a method of steady flow.

The emanation supplied at a constant rate from an actinium preparation was conveyed with a steady stream of air through a tube. The period was deduced from observations of the concentration of the emanation at different points along the tube.

Evidence, which will be discussed later, pointed to the existence of products of yet shorter life; the steady flow method was accordingly adapted to their investigation.

By this method the periods were determined of two products, the one successive to actinium emanation, the other to thorium emanation. The periods were found to be 0020 second and 014 second respectively.

Throughout this paper the new nomenclature proposed by Rutherford and Geiger $\dagger$ will be adopted. To avoid confusion the period will be added in brackets whenever a product is mentioned of which the name has been altered. The two products just mentioned will therefore be called Actinium A (.002 sec.) and Thorium A ( $14 \mathrm{sec}$.$) .$

The steady flow method can be used to investigate any

* Communicated by Prof. E. Rutherford, F.R.S.

+ See preceding paper.

Plil. Mag. S. 6. Vol. 22. No. 130. Oct. 1911. 
product which can be both separated from its parent and brought to an instrument for the measurement of its radiation, provided the time interval is not large compared with the period of the product.

For the separation, advantage was taken * of the fact that the residue after the expulsion of an $\alpha$ particle is positively charged, and will therefore, if driven by recoil into the air, be carried to a negatively charged plate. By using the face of a rotating disk as the negatively charged plate, the separated product was quickly brought to an ionization vessel for the measurement of its radiation.

The period was deduced from the loss of activity of the plate during the time taken to pass from the one to the other of two similar ionization vessels.

The rotating disk used was built for running at high speeds, and was originally used by Schuster and Hemsalech for photographing sparks. Details of the construction will be found in their paper + . It could conveniently be run at a peripheral speed of 160 metres per second.

The disk was turned by an electric motor, and no difficulty was experienced in keeping constant the speed, which was read from a speed counter attached to the hub of the disk. When working with the product thorium A $(0 \cdot 14$ sec.); the speed was reduced by introducing a pair of intermediate wheels between motor and disk.

Preliminary experiments showed that there were no serious difficulties involved in the use of the method. It was accordingly applied to the investigation of the supposed anomalies $\ddagger$ in the decay of the recoil product from the active deposit of actinium.

It was next intended to search for products of short life associated with the emanations of actinium and thorium, the existence of which had been rendered probable by the work of Geiger and Marsden on double scintillations $\$$.

At this stage Geiger \| by a different and ingenious method proved the existence of the new actinium product, and formed an estimate of its period.

We are much indebted to Dr. Geiger for his kindness in leaving to us the further investigation of the period of this product.

The following apparatus was used for investigating the products which follow actinium and thorium emanations.

* Hahn and Meitner, Ber. d. D. Phys. Gesel. vol, xi. p. 55 (1909).

+ Phil. 'Traus. A. rol. cxciii. p. 189 (1899).

I Blanquies, Le Radium, vol. vi. p. 230 (1909).

$\$$ Phys. Zeit. vol, xi. p. 7 (1910).

i| Phil. Mag, vol, xxii. p. 201 (1911). 
The actinium emanation was derived from actinium purified in this laboratory by Prof. Boltwood * from the Royal Sociey residues; the thorium emanation from a preparation of mesothorium. The actinium was wrapped in blotting-paper, throngh which the emanation readily diffused; the mesothorium was contained in a small open tube. Either was fixed inside an ebonite box, $4 \times 3 \times 3 \mathrm{~cm}$, , open at one end, the edges of which, covered with with velvet, pressed against the rotating disk, thus preventing much emanation from escaping. In front of the active material and parallel to the disk was a plate of copper gauze. When the latter was charged positively, the positively charged residue of the emanation was deposited on the face of the disk. Since electroscopes were affected by the vibration caused by the disk when rotating at high speed, ionization boxes and an electrometer were used for measuring the radiation. The two ionization boxes were of similar size and shape. The bottom of each was closed with goldbeater's-skin, which, while impervious to emanation, readily allowed $\alpha$ particles to pass through, since its stopping power was equivalent to less than $2 \mathrm{~cm}$. of air. The goldbeater's-skin was coated on its inner side with aluminium leaf in metallic connexion with the rest of the box. For measuring actinium $A$, the ionization boxes were placed side by side, and the ebonite box containing the actinium was close to them. In the case of thorium $A$, the three boxes were $120^{\circ}$ apart. In either case the boxes were similarly situated with regard to the axis of rotation of the disk, the bottoms of the ionization boxes facing the disk at a distance of about half a centimetre.

The shape of the bottom of an ionization box was that of a truncated sector of the disk, while a brass plate of similar shape, slightly smaller, was insulated inside the box, parallel to the bottom. This plate could be connected to the electrometer by a wire, enclosed in a air-tight metal tube, to guard against induction disturbance and the entrance of emanation. The ionization hoxes were kept charged to a potential of 400 volts, and the current which passed between the box and insulated plate was measured by a compensation method, the plate and connected quadrants of the electrometer being kept during the course of a measurement at zero potential by the use of an induction balance $t$.

In the case of thorium $A$, the thorium and radium emanations-derived from the impure messthorium-escaping from the ebonite box, caused some ionization in the electrometer ;

* Proc. Rov. Soc. A. vol. lxxxv. p. 77 (19ll).

+ Townsend, P'hil. Mag. vol. vi. p. 603 (1908). 
so that serious error due to faulty insulation would have been introduced had the insulated system not been kept at zero potential.

The current was measured by connecting the brass plate to the electrometer for exactly one minute, and noticing both the quantity of electricity used in compensation during that time, and the deflexion of the electrometer-needle due to imperfect compensation.

Measurements were taken in the following order at intervals of two minutes of :-

I. The current through the first box due to $\alpha$ particles from emanation and active deposit on the disk.

II. The current through the first box with a field of +800 volts between the copper gauze and the disk.

III. and IV. Similar measurements in the second box.

In a typical experiment the following currents were observed: 1. 298. II. 402. III. 183. IV. 236. The differences between II. and I. and IV. and III. were evidently due to $\alpha$ particles from actinium $A$ which had been deposited on the disk.

The sensitiveness of the first ionization box was known to be 13 per cent. greater than that of the second.

The ratio of the activities of the actinium $A$ on the disk when opposite the first and second boxes was therefore $104: 53 \times 1 \cdot 13$.

If, now, $\lambda$ is the decay constant of actinium $A$, and $t$ the time taken to pass from the first box to the second

$$
e^{-\lambda t}=\cdot 575 \text {. }
$$

The angular separation of the two boxes was $16^{\circ} \cdot 4$, and the time of one revolution was 037 sec.

Hence $t=\cdot 00169$ sec., giving $\lambda=329$ (sec.) ${ }^{-1}$, and the half-value period $\log _{e} 2 / \lambda=\cdot 00211$ sec.

These measurements were always repeated about five times in the same order. Separate curves were then drawn showing the variation with time of the currents I., II., III., IV., and values of the decay ratio deduced from simultaneous values of the four currents obtained by interpolation. The growth of active deposit and any steady change in the deposition of actinium $\mathrm{A}$ on the disk were in this way allowed for. In some sets of experiments irregular changes took place, due no doubt to small changes in position of the ebonite box, whereby both the amount of actinium emanation escaping, the amount remaining as a source of actinium $\mathrm{A}$, and the amount of activity removed from the disk by the contact of the velvet edging were altered. Such experiments 
were discarded, the probable error in the mean of the various decay ratios obtained from the sets of readings deciding the value of the result.

In this way the following values of $\lambda$ were obtained

\begin{tabular}{llc}
\multicolumn{1}{c}{$t}$. & $\lambda t$. & $\lambda(\mathrm{sec})^{-1}$. \\
.000726 sec. & 0.234 & 323 \\
.000843 & 0.316 & 375 \\
.00169 & 0.562 & 332 \\
.00260 & 0.861 & 332 \\
.00405 & 1.56 & 386
\end{tabular}

Weighted mean $\lambda=347$. Hence half-value period $=\cdot 00200$ sec., the error being probably not greater than 5 per cent.

In fig. 1 the logarithm to base $e$ of the activity $(-\lambda t)$ is plotted against the time $(t)$.

Fig. 1.

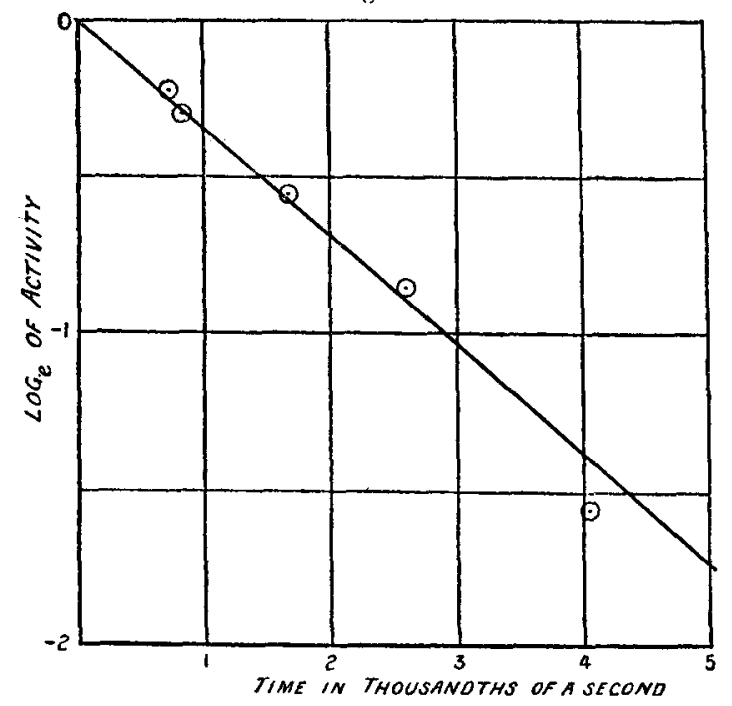

This result is in agreement with that of Geiger * who, from the behaviour of actinium emanation in an electric field, deduced the existence of this new product and the order of magnitude of its period. The value given by Geiger as a rough estimate is, by a remarkable coincidence, identical with that obtained above.

It has been mentioned in the foregoing calculation that the sensitiveness of the first box was found to be 13 per cent.

* Phil. Mag. vol. xxii. p. 201, July 1911. 
greater than that of the second. This was determined by continuing the deposition of actinium $\mathrm{A}$ until by its decay a large quantity of active deposit had been formed on the disk. The actinium was then removed and the currents in the two boxes compared, allowance being made for the decay in activity of the deposit. The observed difference in sensitiveness may in part have been due to imperfect adjustment of the positions of the boxes, so that a little activity may have escaped the second box but not the first. It will be seen that in correcting for sensitiveness, thus determined, error due to faulty adjustment is at the same time eliminated.

In the case of thorium $A$, the correction for sensitiveness was made in an entirely different manner. Sets of experiments were made alternately with the disk rotating in opposite directions, the ratios of the activities then giving $k e^{ \pm \lambda t}$, where $k$ is the sensitiveness ratio. That value of $k$ was then chosen which made the mean value of $\lambda$ from all the "clockwise" experiments the same as the mean from all the "counterclockwise." In other respects the experiments on thorium A were similar to those on actinium $A$, but in the former case the calculation was simpler, since the growth of active deposit. was usually inappreciable. Unexpected irregularities were occasionally observed, but, owing to the departure from England of one of the authors, it was not found possible to inquire into their cause. The mean value found for the half-value period of thorium $A$ was 0.145 second, and this value is thought to be correct to within 10 per cent. A more careful determination will, however, be made as soon as work on this subject is resumed. The result is in agreement with the deduction of Geiger and Marsden*, that an $\alpha$-ray product exists in thorium emanation with a period of the order of a fifth of a second.

\section{Experiments with Actinium $C$.}

The active deposit from actinium is known to consist of three successive products, actinium B ( $36 \mathrm{~min}$.), C ( $2 \cdot 1 \mathrm{~min}$.), and $D(4.7$ min.). Mlle. Blanquies, believing the $\alpha$ radiation of actinium $C$ to be complex $\dagger$, has snggested that it may have come from more than one product, and a product named actinium $\mathrm{B}_{2}$ has obtained a provisional place in Mme. Curie's 'Radio-activity.' 'The results of experiments made by Mlle. Blanquies to test this question $\ddagger$ were not conclusive, but irregularities were observed in the decay of actinium (,,

* Phys. Zeit. vol. xi. p. ? (1910).

† Le Radium, vol. vi. p. 230 (1608).

† Le Liadium, rol, vii. p. 159 (1910). 
and of the product obtained by recoil from the active deposit, which were consistent with the existence of a product subsequent to actinium $\mathrm{C}$, with a period of a few seconds.

The smallness of the observed effect would be explained by the fact that no measurements were taken until a minute after the decay had started.

The existence of this product was therefore tested by the method described in this paper.

A plate rendered active by exposure to actinium emanation was placed in front of the rotating disk. Opposite the disk was an electroscope with an opening large enough to receive a fourth part of any radiation coming from the disk. The electroscope was fixed to a bracket projecting from a wall, in order to avoid the vibration set up by the rotation of the disk.

When the plate was uncharged, no activity was transferred to the disk. On charging the plate positively, those atoms which by the expulsion of an a particle had been projected into the air, were carried to the disk. Such atoms consisted of actinium D and of the hypothetical new product. Since actinium $\mathrm{D}$ does not emit $\alpha$ particles, the ionization in the electroscope would be mainly due to the new product, unless this product either had decayed before reaching the electroscope, or belonged to some side branch from the main disintegration series. The rise of activity on the disk, on

Fig. 2.

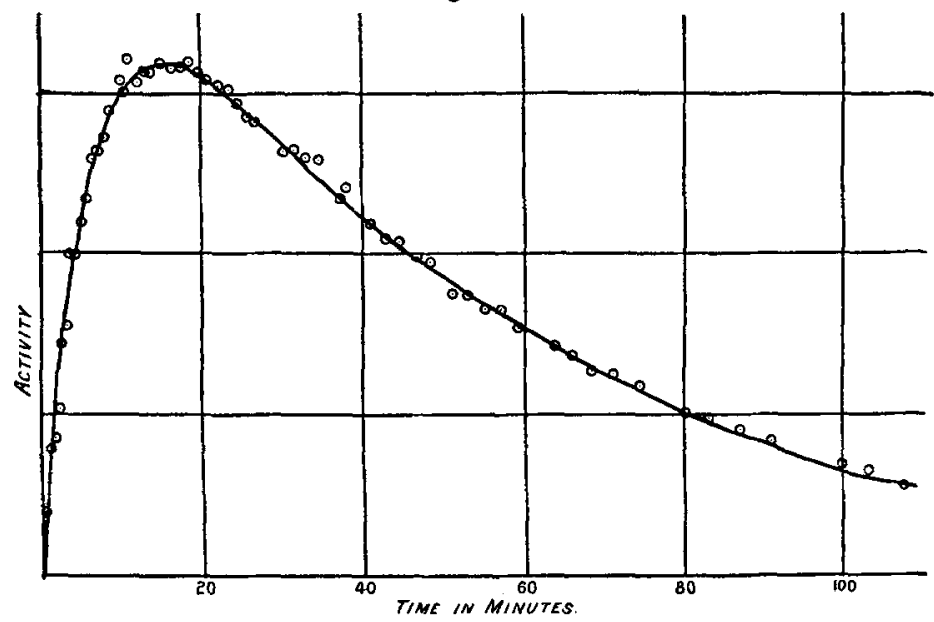

charging the active plate, is shown in fig. 2, the time interval before reaching the electroscope being $\cdot 15$ second. 
The curve drawn has been calculated on the assumption that the recoil product consists entirely of actinium $D$, and the agreement between theory and experiment is seen to be satisfactory.

It is to be remarked that the recoil experiments of Mlle. Blanquies were complicated by the presence in the recoil product of some actinium B. We, on the contrary, always obtained by recoil, either from an active plate or from a fine wire, actinium $\mathrm{D}$ alone.

The presence of a product of short life would also be shown by a rapid initial fall of activity on removing the active source. The rise of activity on the disk was measured as before, until, after $15 \cdot 7$ minutes, the maximum was reached. The active plate was then earthed, and the activity on the disk was found to decay exponentially with the period of actinium D, the activity, immediately after the plate was earthed, being found by extrapolation to be the same as immediately before.

In the ligbt of these experiments, the observation of Geiger and Marsden * that 10 per cent. of the $a$ particles from actinium active deposit are emitted in pairs, seems only to be explained by the existerce after actinium $\mathrm{C}$ of a sidebranch product of very short life. Observations have been made with a small ionization vessel, and the disk rotating at high speed, in which such a product should have been detected had its period been greater than $10^{-4}$ second.

A short description will now be given of a simple method by which products obtained by recoil may be analysed, and products of short life detected. The method is also suited to the examination of the radiation from such products.

The bottom of an electroscope is closed by a sheet of lead, in which is a large hole covered over with aluminium leaf. The active plate, the source of the recoil, is fixed, active side downwards, to the lead sheet.

Any ionization produced in the electroscope is due to $\gamma$ radiation, and to $\beta$ radiation reflected into the opening from surrounding objects.

Directly underneath the opening and in full view of the active plate is placed a small insulated metal plate, or reflector. This reflector being charged to a high negative potential by an influence machine, attracts to itself the product recoiling from the active plate, and the radiation from this product enters the electroscope.

Using actinium active deposit as the source of activity the results shown in fig. 3 were obtained. 
The straight line marked "Diffused Radiation" gives the effect observed when the reflector is uncharged. The upper

Fig. 3.

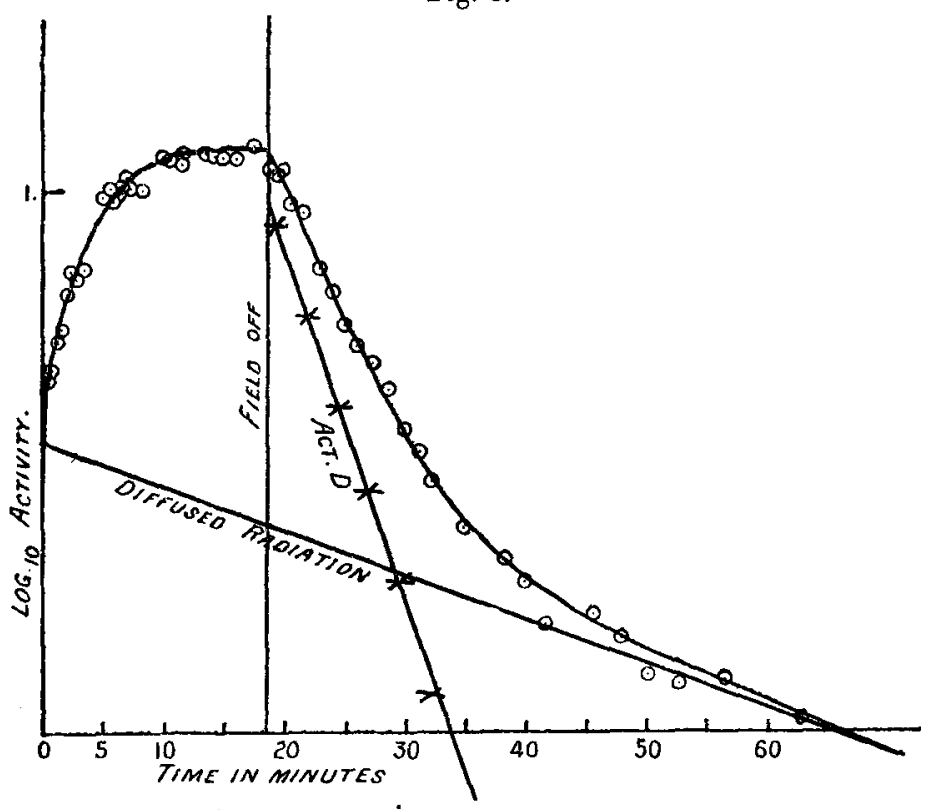

curve was obtained when the reflector was charged by a Wimshurst machine to a jotential of about 10,000 volts, the distance between source and reflector being $5 \mathrm{~cm}$. After 18 minutes the reflector was again earthed. By subtracting the diffused radiation from the latter part of the upper curve, the straight line marked "Act D" was obtained. It wil! be noticed that the two branches of the upper curve meet; a proof that no product of period of a few seconds has been deposited by recoil.

Sensibility of the new method.

A brief discussion will now be given of the possibilities of the new method for detection of products of very short life. The time interval between the formation of a new product and the detection of its effect depends on two factors: (1) the interval required for deposition of the active matter on the rotating disk, (2) the interval between deposition and measurement-which is determined by the angular distance of the detecting vessel from the source and the speed of rotation of the disk. The minimum time lost by a charged 
atom in reaching the rotating disk is limited by the dielectric strength of the air, the velocity ${ }^{*}$ in an electric field of one volt per centimetre being about $1 \cdot 4 \mathrm{~cm}$. per second. The minimum time lost will therefore be about $10^{-5}$ second.

The time lost in reaching the measuring instrument will be least when the product emits a particles, and these are counted by the method of scintillations. Since the distance between source and zinc-sulphide screen need be not more than a few millimetres, measurements may be taken between $10^{-5}$ and $10^{-4}$ second after the separation of the product.

It is intended by this method to extend the investigation into the possible complexity of radium $C$ and of thorium $C$ (period $55 \mathrm{~min}$. ) ; preliminary experiments, in which the loss of time was much greater, having given negative results.

It is evident, however, that for a product of which the parent does not emit a particles, the main difficulty will be in effecting the separation.

It is intended also to use the method for studying the nature of the $\beta$ radiation from radium $\mathrm{C}_{2} \dagger$, the advantage being obvious of working with a source wbich decays to half value with the period of radium $\mathrm{C}, 19.5$ minutes, rather than with that of radium $\mathrm{C}_{2}$ itself, viz. only 1.4 minutes.

$\mathrm{By}$ increasing the size of the ionization vessel, more than half of the activity of the separated product will be available for measurement.

\section{Summary.}

1. Actinium emanation is succeeded by a solid product, which emits a particles, and has a half-value period 0020 second.

2. Thorium emanation is succeeded by a similar product with half-value period $0 \cdot 14$ second.

3 . The product obtained by recoil from actinium active deposit is pure Act $D$. The supposed complex nature of Act $\mathrm{C}$ is therefore not confirmed.

In conclusion we wish to express our sincere gratitude to Professor Rutherford, both for the loan of much radioactive material, and for the kind interest which he has taken in this research.

Physical Laboratory,

University of Manchester.

* Franck, Verhand. d. D. Phys. Gesel. vol. xi. p. 55 (1909).

$\dagger$ Fajans, Phys. Zeit. vol, x. p. 372 (1911). 